\title{
MicroRNAs as future therapeutic targets in COPD?
}

\author{
Ken R. Bracke ${ }^{1}$ and Pieter Mestdagh ${ }^{2,3}$
}

Affiliations: ${ }^{1}$ Dept of Respiratory Medicine, Ghent University Hospital, Ghent, Belgium. ${ }^{2}$ Center for Medical Genetics, Ghent University, Ghent, Belgium. ${ }^{3}$ Cancer Research Institute Ghent, Ghent University, Ghent, Belgium.

Correspondence: Ken R. Bracke, Dept of Respiratory Medicine, Ghent University Hospital, Medical Research Building 2, De Pintelaan 185, B-9000 Ghent, Belgium. E-mail: ken.brackedUUGent.be

@ERSpublications

What obstacles need to be negotiated before microRNA-based therapeutics can be used in COPD? http://ow.ly/fM4n30aD4BX

Cite this article as: Bracke KR, Mestdagh P. MicroRNAs as future therapeutic targets in COPD? Eur Respir J 2017; 49: 1700431 [https://doi.org/10.1183/13993003.00431-2017].

Chronic obstructive pulmonary disease (COPD) represents a major cause of chronic morbidity and mortality, affecting more than 200 million people worldwide and leading to approximately 3 million deaths each year. COPD is mainly caused by cigarette smoking and is characterised by a chronic inflammation leading to obstruction of the small airways and destruction of lung parenchyma (emphysema). Therapies that slow down the accelerated decline in lung function in patients with COPD are still lacking. Therefore, it is essential to unravel the mechanistic processes that underlie the inflammatory reaction and subsequent structural changes in COPD [1].

Aberrant cross-talk between epithelial and mesenchymal cells has been associated with inflammatory and remodelling processes in COPD. Osei et al. [2] previously demonstrated that airway epithelial cells (AECs) from COPD patients release more interleukin (IL)-1 $\alpha$ upon in vitro exposure to cigarette smoke extract. Moreover, using an elegant co-culture model, they demonstrated that this epithelial-derived IL-1 $\alpha$ induced a pro-inflammatory lung fibroblast phenotype, releasing high amounts of the neutrophil attracting chemokine IL-8 [2]. Interestingly, we and others have reported increased levels of IL-1 $\alpha$ in the lungs of patients with COPD and have shown in in vivo cigarette smoke models that neutrophilic inflammation is strongly dependent on IL- $1 \alpha[3,4]$.

In the current issue of the European Respiratory Journal, OsEI et al. [5] demonstrate that the dysfunctional cross-talk between AECs and fibroblasts in COPD is due to the impaired ability of COPD fibroblasts to upregulate microRNA-146a-5p. MicroRNAs (miRNAs) are endogenous, small noncoding RNAs with a regulatory function on gene expression. They bind in a sequence-specific manner to sites with imperfect complementarity in target messenger RNAs (mRNAs), leading to direct inhibition of protein translation or degradation of the transcript. In this way, miRNAs can interact with hundreds of genes simultaneously and regulate several developmental and physiological processes, including cellular proliferation, differentiation, apoptosis and innate and adaptive immune responses [6].

miR-146a-5p has frequently emerged as a regulator of inflammation [7]. Upon activation of several inflammatory pathways, such as Toll-like receptor (TLR) or IL-1R signalling, miR-146a-5p is induced in a nuclear factor (NF)- $\kappa \mathrm{B}$-dependent manner [8]. By targeting key molecules downstream of TLR and IL-1R pathways like tumour necrosis factor receptor-associated factor-6 and IL-1 receptor-associated kinase (IRAK)-1, it functions as a negative feedback regulator, limiting the intensity and duration of the inflammatory response [9]. In their co-cultures of airway epithelial cells and primary lung fibroblasts, OsEI

Received: Feb 282017 | Accepted after revision: March 012017

Conflict of interest: None declared.

Copyright CERS 2017 
et al. [5] demonstrated that epithelial-derived IL-1 $\alpha$ induces miR-146a-5p expression in fibroblasts. Importantly, this increase of miR-146a-5p was impaired in fibroblasts from patients with COPD, which releases the brake on the IL-1R/NF- $\kappa \mathrm{B}$ pathway and is likely to contribute to the abnormal inflammation in COPD. Interestingly, treating fibroblasts with an miR-146a-5p mimic downregulated the expression of IRAK-1, resulting in a reduced release of IL-8 and confirming the anti-inflammatory role of miR-146a-5p [5]. It is worth noting that the inability of COPD fibroblasts to upregulate miR-146a-5p not only leads to an impaired negative feedback regulation of NF- $\kappa \mathrm{B}$ signalling, but also results in a reduced mRNA degradation and thus prolonged half-life of cyclooxygenase (COX)-2, another target of miR-146a-5p [10]. COX-2 is a key enzyme in biosynthesis of prostaglandin $\mathrm{E}_{2}$, a promoter of neutrophil recruitment and an inhibitor of the repair function of fibroblasts.

In an attempt to find the mechanism explaining the inability of COPD lung fibroblasts to upregulate miR-146a-5p, OsEI et al. [5] assessed the presence of the single nucleotide polymorphism (SNP) rs2910164 in their primary lung fibroblasts. This common G>C SNP in pre-miR-146a-5p has been associated with decreased expression of the mature miR-146a-5p [11]. Unexpectedly, in the present study, a lower miR-146a-5p expression was observed in fibroblasts from donors homozygous for the $G$ allele of SNP rs2910164, which were all but one COPD patient [5]. A validation study in a large independent cohort will be needed to verify these results. Moreover, despite the strong association of this SNP with the presence of COPD, no such association was found in any of the large genome-wide association studies of lung-function parameters of airflow obstruction (i.e. forced expiratory volume in $1 \mathrm{~s}$ or its ratio to forced vital capacity) [12].

Since miRNAs are key regulators of the human transcriptome and are differentially expressed in nearly every disease, there is an enormous therapeutic potential in both miRNA inhibitors and mimics. In recent years, several miRNA therapeutics have gone into preclinical development and have been used in clinical trials [13]. To date, the most clinically advanced miRNA therapy is miravirsen, a locked nucleic acid modified miR-122 inhibitor used for the treatment of hepatitis C virus (HVC) infections [14]. miR-122 is a liver-specific miRNA and is required for the propagation of HCV. Phase II clinical trials with miravirsen reported a significant, dose-dependent decrease in HCV, which sustained long after the administration [14], demonstrating the effectiveness of miRNA therapy in humans.

Whether or not miRNA-based therapeutics will ever be used for the treatment of such a complex and heterogeneous disease as COPD depends on the hurdles that still lie ahead. First, we need a clear picture

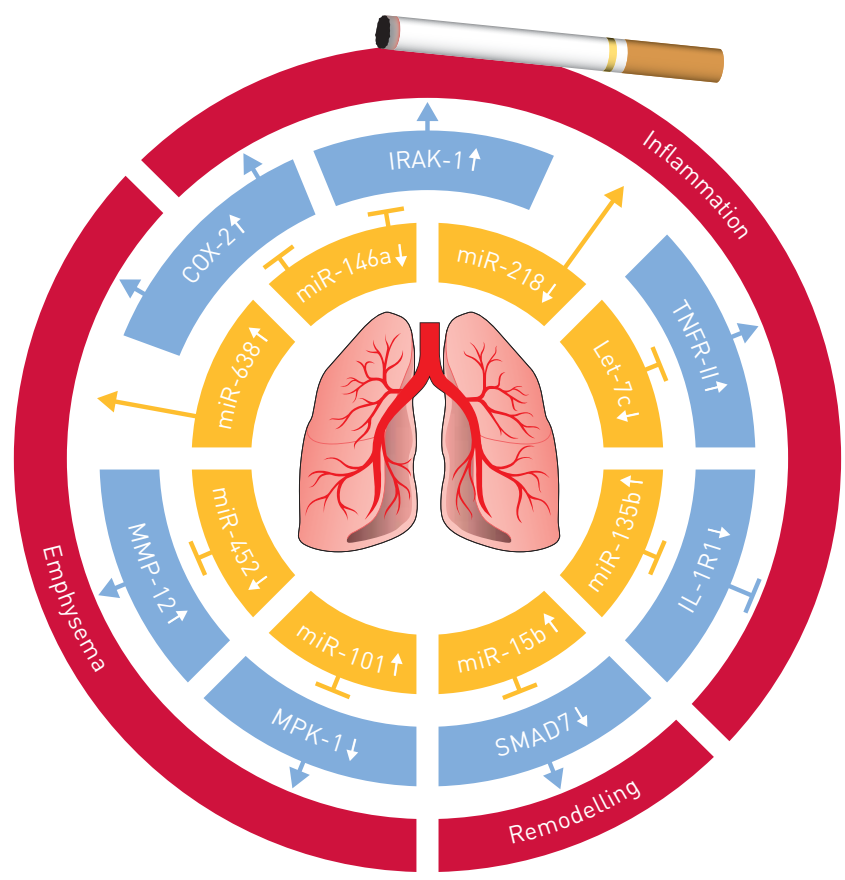

FIGURE 1 Biological functions of microRNAs (miRNAs) implicated in the pathogenesis of chronic obstructive pulmonary disease (COPD). Simplified scheme of miRNAs that have been implicated, by in vitro or in vivo functional studies, in several hallmarks of COPD, including chronic inflammation, airway remodelling and emphysema. This illustration highlights the possible contribution of miR-146a [5, 10], miR-218 [20], let-7c [18], miR-135b [21], miR-15b [15], miR-101 [22], miR-452 [17] and miR-638 [23] to the pathogenesis of COPD. COX: cyclooxygenase; IRAK: interleukin-1 receptor-associated kinase; TNFR: tumour necrosis factor receptor; IL: interleukin; MPK: mitogen-activated protein kinase; MMP: matrix metalloproteinase. 
of the candidate miRNAs. In the last decade, several miRNA profiling studies have been published, yielding lists of miRNAs that are differentially expressed between control subjects and smokers with or without COPD [15-17]. Unfortunately, large discrepancies exist between the different studies. These can be due to differences in sample material, ranging from whole lung tissue to epithelial brushings and induced sputum. We were the first to show a significant differential expression of miRNAs in the induced sputum of patients with COPD and current smokers compared with never-smokers, including downregulation of miR-146a-5p [18]. Next to sample material, the characteristics of the study population (including chronic bronchitis versus emphysematous phenotype, COPD severity, smoking history, age, sex) may also substantially influence the outcome. And finally, the different platforms used for miRNA profiling, ranging from microarrays to RT-qPCR and, the current gold standard, small RNA sequencing, do not always deliver the same results [19].

Once differentially expressed miRNAs have been identified, the next step is to validate the biological significance of these findings. In vitro and in vivo functional studies have already implicated several miRNAs in the pathogenesis of COPD (figure 1) [24, 25]. Recently, using in vivo perturbation experiments and gene set-enrichment analysis, we were able to demonstrate a protective role of miR-218-5p in cigarette smoke-induced inflammatory responses and COPD [20]. Importantly, miR-218-5p is significantly down-regulated in bronchial epithelium of patients with COPD [20]. Translation of these in vitro and animal studies to humans will require substantial validation and mechanistic studies, with an important focus on the dosing of miRNA therapy. Many of the functional studies, including the current study by Osei et al. [5], use doses of miRNA inhibitors or mimics that are beyond physiological relevance. Additionally, it seems likely that miRNA-based therapy for a complex disease like COPD, encompassing several immunological pathways and cell types, will have to be directed at multiple miRNAs. Moreover, the interplay between different miRNAs, which may share several targets, should be taken into account.

Another hurdle is the delivery of the miRNA inhibitors or mimics. Targeting of miRNA-based therapeutics to a particular tissue or specific cell type is a challenging area of research [26]. It seems obvious that, for example, targeting the airway epithelium is more feasible than the underlying fibroblasts. Therefore, the route of administration (local versus systemic) as well as the delivery method (from viral or lipid vectors to nanoparticle and polymer systems) should be considered carefully. Importantly, nonspecific targeting of miRNA therapeutics may lead to toxicity. Indeed, while the ability of miRNAs to target multiple mRNAs and pathways is key to their enormous therapeutic potential, it may also be a limitation, giving rise to unwanted effects in other cell types.

Taken together, profiling studies have yielded several miRNAs that are dysregulated in COPD. The need for functional studies like the current work by OsEI et al. [5] is great, for demonstration of the biological relevance of these candidate miRNAs. Ideally, to establish miRNAs as promising therapeutic targets in COPD, in vivo studies should be performed, taking into account physiologically relevant dosing and targeted delivery to the desired lung compartment or cell type.

\section{References}

1 Brusselle GG, Joos GF, Bracke KR. New insights into the immunology of chronic obstructive pulmonary disease. Lancet 2011; 378: 1015-1026.

2 Osei ET, Noordhoek JA, Hackett TL, et al. Interleukin-1alpha drives the dysfunctional cross-talk of the airway epithelium and lung fibroblasts in COPD. Eur Respir J 2016; 48: 359-369.

3 Pauwels NS, Bracke KR, Dupont LL, et al. Role of IL-1alpha and the Nlrp3/caspase-1/IL-1beta axis in cigarette smoke-induced pulmonary inflammation and COPD. Eur Respir J 2011; 38: 1019-1028.

4 Botelho FM, Bauer CM, Finch D, et al. IL-1alpha/IL-1R1 expression in chronic obstructive pulmonary disease and mechanistic relevance to smoke-induced neutrophilia in mice. PLoS One 2011; 6: e28457.

5 Osei ET, Florez-Sampedro L, Tasena H, et al. miR-146a-5p plays an essential role in the aberrant epithelialfibroblast cross-talk in COPD. Eur Respir J 2017; 49: 1602538.

Bartel DP. MicroRNAs: genomics, biogenesis, mechanism, and function. Cell 2004; 116: 281-297.

Rusca N, Monticelli S. MiR-146a in immunity and disease. Mol Biol Int 2011; 2011: 437301.

8 Perry MM, Williams AE, Tsitsiou E, et al. Divergent intracellular pathways regulate interleukin-1beta-induced miR-146a and miR-146b expression and chemokine release in human alveolar epithelial cells. FEBS Lett 2009; 583: 3349-3355.

9 Taganov KD, Boldin MP, Chang KJ, et al. NF-kappaB-dependent induction of microRNA miR-146, an inhibitor targeted to signaling proteins of innate immune responses. Proc Natl Acad Sci USA 2006; 103: 12481-12486.

10 Sato T, Liu X, Nelson A, et al. Reduced miR-146a increases prostaglandin E(2)in chronic obstructive pulmonary disease fibroblasts. Am J Respir Crit Care Med 2010; 182: 1020-1029.

11 Jazdzewski K, Murray EL, Franssila K, et al. Common SNP in pre-miR-146a decreases mature miR expression and predisposes to papillary thyroid carcinoma. Proc Natl Acad Sci USA 2008; 105: 7269-7274.

12 Hancock DB, Eijgelsheim M, Wilk JB, et al. Meta-analyses of genome-wide association studies identify multiple loci associated with pulmonary function. Nat Genet 2010; 42: 45-52.

13 Christopher AF, Kaur RP, Kaur G, et al. MicroRNA therapeutics: discovering novel targets and developing specific therapy. Perspect Clin Res 2016; 7: 68-74. 
14 Janssen HL, Reesink HW, Lawitz EJ, et al. Treatment of HCV infection by targeting microRNA. $N$ Engl J Med 2013; 368: 1685-1694.

15 Ezzie ME, Crawford M, Cho JH, et al. Gene expression networks in COPD: microRNA and mRNA regulation. Thorax 2012; 67: 122-131.

16 Schembri F, Sridhar S, Perdomo C, et al. MicroRNAs as modulators of smoking-induced gene expression changes in human airway epithelium. Proc Natl Acad Sci USA 2009; 106: 2319-2324.

17 Graff JW, Powers LS, Dickson AM, et al. Cigarette smoking decreases global microRNA expression in human alveolar macrophages. PLoS One 2012; 7: e44066.

18 Van Pottelberge GR, Mestdagh P, Bracke KR, et al. MicroRNA expression in induced sputum of smokers and patients with chronic obstructive pulmonary disease. Am J Respir Crit Care Med 2011; 183: 898-906.

19 Mestdagh P, Hartmann N, Baeriswyl L, et al. Evaluation of quantitative miRNA expression platforms in the microRNA quality control (miRQC) study. Nat Methods 2014; 11: 809-815.

20 Conickx G, Mestdagh P, Avila Cobos F, et al. MicroRNA profiling reveals a role for microRNA-218-5p in the pathogenesis of chronic obstructive pulmonary disease. Am J Respir Crit Care Med 2017; 195: 43-56.

21 Halappanavar S, Nikota J, Wu D, et al. IL-1 receptor regulates microRNA-135b expression in a negative feedback mechanism during cigarette smoke-induced inflammation. J Immunol 2013; 190: 3679-3686.

22 Hassan F, Nuovo GJ, Crawford M, et al. MiR-101 and miR-144 regulate the expression of the CFTR chloride channel in the lung. PLoS One 2012; 7: e50837.

23 Christenson SA, Brandsma CA, Campbell JD, et al. miR-638 regulates gene expression networks associated with emphysematous lung destruction. Genome Med 2013; 5: 114

24 De Smet EG, Mestdagh P, Vandesompele J, et al. Non-coding RNAs in the pathogenesis of COPD. Thorax 2015; 70: 782-791.

25 Osei ET, Florez-Sampedro L, Timens W, et al. Unravelling the complexity of COPD by microRNAs: it's a small world after all. Eur Respir J 2015; 46: 807-818.

26 Simonson B, Das S. MicroRNA therapeutics: the next magic bullet? Mini Rev Med Chem 2015; 15: 467-474. 